\title{
Análisis de los ingresos ginecoobstétricos a Terapia Intensiva de un hospital de Matamoros, Tamaulipas, de 2012-2018
}

Analysis of gyneco-obstetric admissions to Intensive Therapy of hospital of Matamoros, Tamaulipas, 2012-2018 Análise das internações ginecológicas e obstétricas em Terapia Intensiva do Hospital de Matamoros, Tamaulipas 2012-2018

\author{
Sol Zenith González Mendoza, ${ }^{*, \neq}$ Jesús Adrián Maldonado Mancillas, ${ }^{*, \ddagger}$ Mario Alberto Tinoco Alvares, ${ }^{*}$ \\ Rodolfo Sergio De la Garza Longoria ${ }^{\ddagger}$
}

\section{RESUMEN}

Introducción: En todo el mundo, se realizan estudios para conocer la casuística de la morbimortalidad ginecoobstétrica, y México no es la excepción: en 2009, Briones reportó las cuatro principales alteraciones que condicionan a la mortalidad en la Unidad de Cuidados Intensivos Obstétricos; y en 2011, Guerrero realizó un estudio con 333 pacientes que hubieron ingresado a la Unidad de Cuidados Intensivos del Hospital General de México. Con base en lo anterior, nosotros establecimos la siguiente pregunta de investigación: ¿cuál será la casuística de las pacientes con complicaciones de ginecoobstetricia que ingresaron a la Unidad de Cuidados Intensivos del Hospital General de Matamoros, Tamaulipas, entre 2012 y 2018 ?

Material y métodos: Con los datos diarios de ingresos y egresos de la Unidad de Cuidados Intensivos, se capturó cada variable, analizando cada una de ellas mediante la forma observacional, retrospectiva, transversal y bajo la técnica de análisis estadístico.

Resultados: De los 2,497 registros ( $n=227$ ), 206 fueron obstétricos complicados; 55 adolescentes estuvieron embarazadas, predominando la eclampsia. De parte de ginecología, se registraron 21 pacientes.

Conclusiones: La casuística es diferente a la publicada en México. Independientemente de esto, se requiere promover los programas de apoyo de la Secretaría de Salud, con la inclusión de instituciones universitarias para que participen los jóvenes y para que cursen programas académicos relacionados con la salud, como la de médico cirujano y enfermería general.

Palabras clave: Ginecoobstetricia, cuidados intensivos, incidencia.

\section{ABSTRACT}

Introduction: Progress in the diagnosis, prevention and casuistry of disorders in pregnancy has produced a significant decrease in maternal morbidity and mortality. Research that aims to understand the number of gyneco-obstetric morbidity cases is conducted in many countries, with Mexico not being the exception. In 2009, Briones reported the four major causes that condition morbidity-mortality in a obstetric Intensive Care Unit (ICU). In 2011, Guerrero conducted a study consisting of 333 patients admitted to the Intensive Care Unit of the General Hospital of Mexico. Therefore, we aim to answer the following question: what is the number of gyneco-obstetric complicated patients that were admitted to the ICU of the General Hospital in Matamoros, Tamaulipas, during the years of 2012 to 2018?

Material and methods: With the journal of income and expenditures of the ICU, each variable is captured; each of them is analyzed; through observational, retrospective and cross-sectional forms and under the statistical analysis technique. Results: Of the 2,497 cases ( $n=227$ ), 206 were complicated obstetrics; 55 cases were adolescent pregnancies; eclampsia prevailing. There were 21 gynecology patients.

Conclusions: The casuistry differs to the one published in Mexico. Regardless it is required to increase and promote programs that support the Ministry of Health, such as the inclusion of universities, and of young people who attend health-related academic programs, such as medical surgeon and nursing; and allow an approach to secondary, preparatory or general community, to address the issues of pregnancy and complications.

Keywords: Obstetrics gynecology, intensive care, incidence.

\section{RESUMO}

Introduçao: No mundo são realizados estudos para determinar a casuística da morbimortalidade ginecobstétrica e o México não é exceção. Em 2009,

\footnotetext{
* Hospital General de Matamoros. Tamaulipas.

‡ Universidad Autónoma de Tamaulipas. Ciudad Victoria, Tamaulipas.
}

Recepción: 16/07/2019. Aceptación: 02/12/2019.

www.medigraphic.com/medicinacritica
Briones relatou as quatro principais alterações que determinam a mortalidade na terapia intensiva obstétrica. Em 2011, Guerrero realizou-se um estudo com 333 pacientes que ingressaram na Unidade de Terapia Intensiva do Hospital Geral do México. Diante do exposto, temos a questão de pesquisa: qual será a casuística de pacientes ginecoobstétricas e complicadas admitidas na UTI do Hospital Geral de Matamoros Tamaulipas nos anos de 2012 a 2018?

Material e métodos: Cada variável é capturada no diário de ingressos e egressos da UCI; analisando cada um deles; através da forma observacional, retrospectiva, transversal e sob a técnica de análise estatística.

Resultados: Das 2,497 ( $n=227), 206$ eram obstetricas complicados; 55 adolescentes gestantes; predominando a eclampsia. De ginecologia foram 21 pacientes.

Conclusões: A casuística é diferente da publicada no México. Independentemente, é necessário promover os programas de apoio à Secretaria de Saúde, com a inclusão de instituições universitárias; e que participem os jovens que frequentam programas acadêmicos relacionados à saúde, como cirurgião e/ou enfermagem Geral. Palavras-chave: Ginecologia e obstetrícia, cuidados intensivos, incidência.

\section{INTRODUCCIÓN}

Cada año, más de cinco millones de estadounidenses ingresan a las Unidades de Cuidados Intensivos (UCI) con enfermedades graves. Gracias a que los avances tecnológicos y médicos han aumentado, la proporción de pacientes que se recupera de una enfermedad crítica es mayor, lo que significa que también se convierten en sobrevivientes de la UCl. ${ }^{1}$

En este sentido, el progreso en el diagnóstico, la prevención de los trastornos en el embarazo y la identificación de los parámetros fetales, los cuales podrían inducir una distocia grave, han producido una disminución significativa de la mortalidad materna; sin embargo, no se tiene una disminución que coincida con la de la morbilidad puerperal y con el número de ingresos en cuidados intensivos. ${ }^{2-4}$

En los países occidentales donde se ha obtenido una disminución de la mortalidad materna, la morbilidad puerperal se ha convertido en el principal tema de investigación, en especial si se considera lo suficientemente grave como para requerir el ingreso a la Unidad de Cuidados Intensivos. Según los criterios de la Federación Internacional de Ginecología y Obstetricia (FIGO), la muerte materna se define como aquella que sucede entre el embarazo y los 42 días posteriores a la finalización de éste, y ahora es considerada un indicador de calidad en la asistencia de salud.

En México, se realizan grandes esfuerzos por tener el control estadístico de la muerte materna ${ }^{5}$ mediante estadísticas vitales, la estimación indirecta de la mortalidad 
materna y las investigaciones acerca de la mortalidad de las mujeres en edad reproductiva, pero ninguna de ellas logra por sí sola un panorama preciso y completo de la mortalidad materna. Y no se puede soslayar el gran trabajo realizado por Fajardo y su equipo de investigación, ${ }^{6}$ en 2009, donde se revisaron 173 expedientes. En este importante trabajo, analizaron cada una de las condiciones médicas que llevan al deceso de las pacientes. Desde el 2001, Loverro ha propuesto llevar a cabo una definición de «transferencia puerperal» hacia la Unidad de Cuidados Intensivos en pacientes complicadas con embarazo o después del parto. Él comenta que existe poca información acerca de datos que estudian la incidencia, las causas y el resultado de la población ginecoobstétrica que requiere atención en cuidados intensivos. ${ }^{7}$ En 1997, en Canadá, se encontró que tan sólo el 0.26\% de la población ginecoobstétrica complicada era transferida a las Unidades de Cuidados Intensivos, ${ }^{8}$ y en estudios europeos, que esta cifra abarcaba sólo el $0.12 \%,{ }^{9}$ predominando en estos estudios algunas coincidencias, como las mujeres jóvenes (edad media de 30 años), que eran primíparas y que ingresaban con una grave complicación del embarazo como la principal causa. En México, en el año 2002, se encontró que las principales causas de morbilidad y mortalidad durante el embarazo eran preeclampsia-eclampsia, hemorragias obstétricas y complicaciones del puerperio, lo que condicionaba al $62 \%$ del total de las muertes maternas, quedando el síndrome HELLP (Hemolysis, Elevated Liver enzymes, and Low Platelets) como la causa de mortalidad entre 5 y $25 \%$, en embarazos menores de 36 semanas. ${ }^{10}$

En estudios realizados por el Departamento de Obstetricia-Ginecología de la University Center Hospital, en Yaundé, Camerún, en 2014, determinaron que más de 550,000 mujeres mueren anualmente en el mundo por causas relacionadas con el embarazo y que $50 \%$ de la estimación mundial de muertes maternas ocurre sólo en África subsahariana. El estudio realizado fue desde el primero de enero de 2006 hasta el 31 de diciembre de 2010 , y las causas de muerte fueron: hemorragia postparto (29.2\%), aborto inseguro (25\%), embarazo ectópico $(12.5 \%)$, hipertensión en el embarazo $(8.3 \%)$, malaria $(8.3 \%)$, anemia (8.3\%), enfermedad cardiaca $(4.2 \%)$, neumonía $(4.2 \%)$ y placenta previa $(4.2 \%)$. Las edades oscilaron entre los 18 y los 41 años, con una media de $27.7 \pm 5.14$. La falta de atención prenatal fue un factor de riesgo para la muerte materna, y la falta de atención prenatal fue un factor de riesgo para la mortalidad materna. ${ }^{11}$

En Pakistán, el Hospital Civil de Karachi del Departamento de Obstetricia y Ginecología Unidad II realizó un estudio desde marzo de 2015 hasta marzo de 2017 , y de los 7,968 partos en dos años, registraron que 32 pacientes se sometieron a histerectomía obstétrica de emergencia (EOH); la edad media fue de $30.0 \pm 5.2$ años; la mayoría de las mujeres $(75 \%)$ fueron multípa- ras. En 10 pacientes (30\%), se realizó $\mathrm{EOH}$ por una hemorragia incontrolable debida a atonía uterina, seguida de una placenta con adherencia mórbida (28\%). La morbilidad incluyó la coagulación intravascular diseminada (CID) en cuatro pacientes (19\%) y tres pacientes se sometieron a laparotomía repetida por hemorragia incontrolable. Hubo tres muertes maternas, con un índice de casos fatales de $9.3 \%$. Los autores concluyeron que el aumento de la tasa de cesáreas era responsable del aumento de la incidencia de $\mathrm{EOH}$, lo que daba como resultado placentas adherentes. ${ }^{12}$

En México, en el 2009, Briones reportó las cuatro principales causas de morbilidad que condicionan mortalidad en una Unidad de Cuidados Intensivos Obstétricos en el periodo comprendido de 1997 a 2007; la primera fue la sepsis en $2.42 \%$ de los casos. ${ }^{13}$ En 2011 , Guerrero realizó un estudio de los pacientes que ingresaron a la Unidad de Cuidados Intensivos, en la Unidad 112 del Hospital General de México. De los 333 pacientes, 46 fueron ginecológicas: por sepsis, choque hipovolémico y apoyo con ventilación mecánica, y 287 obstétricas: preeclampsia, hemorragia, sepsis y otras patologías. ${ }^{14}$ Por lo antes mencionado, e inspirados en las investigaciones similares y llevadas a cabo en nuestro país, la presente investigación se realizó con el objetivo de conocer cómo se encuentra la casuística de Terapia Intensiva e Ingresos de Ginecología y Obstetricia de nuestro hospital en comparación con los del resto del país y, a través de ello, contribuir en el conocimiento de cómo se comporta la atención médica en nuestra localidad. Bajo un enfoque metodológico, también se busca conocer la información básica de las pacientes de ginecología y obstetricia que entran al Servicio de Unidad de Cuidados Intensivos al Hospital General de Matamoros, Tamaulipas, y que mediante ello se pudiese explicar las causas relacionados con esta incidencia. Por tanto, se tomó como pregunta de investigación: ¿cuál será la casuística de pacientes de ginecoobstetricias con complicaciones que ingresaron a la Unidad de Cuidados Intensivos del Hospital General de Matamoros, Tamaulipas, entre 2012 y 2018 ?

\section{MATERIAL Y MÉTODOS}

Bajo la aprobación del Comité de Investigación y Bioética, así como de la alta dirección del Hospital General de Matamoros, Tamaulipas, en la presente investigación se utilizaron dos instrumentos: el primero fue con el libro de registro de ingresos y egresos de la Unidad de Cuidados Intensivos del Hospital General de Matamoros, y el segundo con el expediente clínico hospitalario de cada paciente.

A partir de un enfoque de tipo observacional, retrospectivo, transversal y bajo la técnica de análisis estadístico, se tomaron como criterios de inclusión todas las 
pacientes adolescentes (hasta la edad de 17 años, 11 meses, 29 días) que llegaron a la $\mathrm{UCl}$, bajo diagnósticos de embarazo complicado y/o con puerperio patológico; con edad de 17 años y 11 meses. Como criterios de exclusión se consideraron aquellos embarazos ingresados a la $\mathrm{UCl}$ en donde las pacientes tuvieran 18 años o más.

\section{RESULTADOS}

De los 2,497 pacientes recibidos en terapia intensiva del Hospital General de Matamoros, Tamaulipas, entre enero de 2012 y diciembre de 2018 , el $8.45 \%(n=211)$ de los casos los recibió el Departamento de Ginecología y Obstetricia. De esta cifra, el 7.6\% (190 pacientes) se presentó con el diagnóstico de embarazo complicado, con edades de los 13 a los 46 años. Cabe mencionar que un total de $2.20 \%$ (55 pacientes) de los embarazos complicados correspondieron a menores de edad (17 años 11 meses).

Se realizó una evaluación de las pacientes jóvenes usando el programa SPSS 25.0. De esta manera, se encontró una correlación bilateral de Rho de Spearman con referente a la edad y el total de pacientes de 0.013 (significativa bilateral arriba de 0.05) (Tabla 1). En cuanto a los diagnósticos encontrados desde el aspecto obstétrico y de complicaciones, llama la atención que los trastornos metabólicos relacionados con la diabetes mellitus descompensada I y II ocupó el menor índice de ingresos con sólo $0.9 \%$ (2 pacientes); en contraste, la eclampsia que concentra el mayor índice del total, con un $34.1 \%$ (72 casos), y en segundo lugar, la preeclampsia con 62 pacientes $(29.4 \%)$. Desde el aspecto ginecológico, encontramos sólo 10\% de los casos (21 pacientes) (Tabla 2). Además, hallamos que la máxima estancia de hospitalización en la UCl fue de una semana (0.5\%), cuando el mayor flujo de movilidad representa sólo 24 horas de estancias con $40.3 \%$ de los casos (Tabla 3). En relación con el motivo de egreso, el estudio arrojó que sólo 2.8\% (6 casos) fue defunción, de los cuales sólo dos casos se relacionan con embarazo complicado y los cuatro restantes fueron de ginecología (Tabla 4). En cuanto al uso de ventilación mecánica, encontramos que 10.9\% (23 pacientes) utilizaron ventilación mecánica y sedación (Tabla 5).

\section{DISCUSIÓN}

De los resultados encontrados por nuestro hospital del Sector Salud, el cual atiende en un segundo nivel, se decidió utilizar la división de pacientes en obstétricos y en ginecológicos, tal como lo propone Guerrero en el 2011, por lo práctico que esto resulta. ${ }^{14}$

Acorde con el estudio encontrado en 2014 , se estableció que los embarazos en adolescentes se están adelantando en el inicio de la vida sexual. ${ }^{15}$ Asimismo, se detectó una diferencia en comparación con los estudios multicéntricos realizados en nuestro país. Cabe señalar que la casuística fue diferente, ya que de los 2,497 ingresos a la Unidad de Cuidados Intensivos, en general, sólo correspondieron a 227 casos para el Servicio de Ginecología y Obstetricia y, de éstos, sólo 55 casos correspondieron a adolescentes embarazadas y complicadas. Sin embargo, en nuestra localidad se aprecia diferente el resultado de los diagnósticos encontrados en comparación con el resto de las investigaciones, ya que tiende a aumentar los procesos de eclampsia con $34.1 \%$ de los casos, pues ésta tiende

Tabla 1: Correlación edad/total del paciente.

\begin{tabular}{|c|c|c|c|c|}
\hline & & & Menores & Total \\
\hline \multirow[t]{6}{*}{$\begin{array}{l}\text { Rho de } \\
\text { Spearman }\end{array}$} & Menores & $\begin{array}{l}\text { Coeficiente de } \\
\text { correlación }\end{array}$ & 1.000 & $0.782^{*}$ \\
\hline & & Sig. (bilateral) & 0.05 & 0.013 \\
\hline & & $\mathrm{n}$ & 9 & 9 \\
\hline & Total & $\begin{array}{l}\text { Coeficiente de } \\
\text { correlación }\end{array}$ & $0.782^{*}$ & 1.000 \\
\hline & & Sig. (bilateral) & 0.013 & \\
\hline & & $\mathrm{n}$ & 9 & 9 \\
\hline
\end{tabular}

* La correlación es significativa en el nivel 0.05 (bilateral).

Tabla 2: Diagnósticos de ingreso a Unidad de Cuidados Intensivos.

\begin{tabular}{lrrrc}
\hline Válido & \multicolumn{1}{c}{$\mathrm{n}$} & \multicolumn{1}{c}{$\%$} & \% válido & \% acumulado \\
\hline Eclampsia & 72 & 34.1 & 34.1 & 34.1 \\
Preeclampsia & 62 & 29.4 & 29.4 & 63.5 \\
Neumopatías/ & 15 & 7.1 & 7.1 & 70.6 \\
gestación & & & & \\
HELLP/gestación & 12 & 5.7 & 5.7 & 76.3 \\
DM-I y Il/gestación & 2 & 0.9 & 0.9 & 77.3 \\
Shock/gestación & 27 & 12.8 & 12.8 & 90.0 \\
Ginecológico & 21 & 10.0 & 10.0 & 100.0 \\
Total & 211 & 100.0 & 100.0 & \\
\hline
\end{tabular}

Tabla 3: Días de estancia en cuidados intensivos.

\begin{tabular}{crrrc}
\hline Día/válido & $\mathrm{n}$ & \multicolumn{1}{c}{$\%$} & \% válido & \% acumulado \\
\hline 0 & 14 & 6.6 & 7.3 & 7.3 \\
1 & 77 & 36.5 & 40.3 & 47.6 \\
2 & 60 & 28.4 & 31.4 & 79.1 \\
3 & 17 & 8.1 & 8.9 & 88.0 \\
4 & 11 & 5.2 & 5.8 & 93.7 \\
5 & 5 & 2.4 & 2.6 & 96.3 \\
6 & 2 & 0.9 & 1.0 & 97.4 \\
7 & 1 & 0.5 & 0.5 & 97.9 \\
8 & 1 & 0.5 & 0.5 & 98.4 \\
11 & 1 & 0.5 & 0.5 & 99.0 \\
12 & 1 & 0.5 & 0.5 & 99.5 \\
25 & 1 & 0.5 & 0.5 & 100.0 \\
Total & 191 & 90.5 & 100.0 & \\
Perdidos por & 20 & 9.5 & & \\
el sistema & & & & \\
Total & 211 & 100.0 & & \\
\hline
\end{tabular}


Tabla 4: Motivo de egreso de cuidados intensivos.

\begin{tabular}{lrrrc}
\hline Válido & \multicolumn{1}{c}{$\mathrm{n}$} & \multicolumn{1}{c}{$\%$} & \% válido & \% acumulado \\
\hline Defunción & 6 & 2.8 & 2.8 & 2.8 \\
Mejoría & 205 & 97.2 & 97.2 & 100.0 \\
Total & 211 & 100.0 & 100.0 & \\
\hline
\end{tabular}

Tabla 5: Ventilación mecánica utilizada en cuidados intensivos.

\begin{tabular}{lrrrc}
\hline Válido & \multicolumn{1}{c}{$\mathrm{n}$} & \multicolumn{1}{c}{$\%$} & \% válido & \% acumulado \\
\hline Sí & 23 & 10.9 & 11.0 & 11.0 \\
No & 186 & 88.2 & 89.0 & 100.0 \\
Total & 209 & 99.1 & 100.0 & \\
Perdidos por & 2 & 0.9 & & \\
el sistema & & & & \\
Total & 211 & 100.0 & & \\
\hline
\end{tabular}

a incidir en los estudios multicéntricos. ${ }^{16}$ Aun así, se destaca una similitud en relación con los trastornos hematológicos y de daño a otros órganos, establecido por hemólisis, elevación de enzimas hepáticas y conteo bajo de plaquetas (por sus siglas en inglés se conoce como síndrome de HELLP) con $5.70 \%$.

Es importante comentar que en los estudios nacionales no determinan con exactitud si el grado de complicación requirió asistencia ventilatoria, pero en nuestro estudio se encuentra que $10.9 \%$ de las pacientes sí necesitó apoyo ventilatorio; desafortunadamente, no tenemos un punto de referencia en cuanto a la estadística nacional.

\section{CONCLUSIÓN}

Dada la dimensión encontrada en el presente estudio, coincidimos en el planteamiento de realizar estrategias como lo que propone Gayet $2014 ;{ }^{15}$ es necesario incrementar y promover los programas mismos, apoyando a la Secretaría de Salud, para que incluya participación directa de instituciones universitarias, y mediante el apoyo de los estudiantes que cursen programas académicos relacionados con la salud comunitaria, tales como la Carrera de Médico Cirujano, Enfermería General, Psicología y Trabajo Social, que permitan acercarse a los derechohabientes que esperan en consulta externa; para que mediante pláticas breves hagan del conocimiento a los pacientes sobre el autocuidado y vigilancia de signos de alarma de embarazo complicado; lo anterior se suma a la vinculación interinstitucional de las universidades y Sector salud en beneficio de la participación a la comunidad. Es importante atender la formación de Recursos Humanos, y efectuar promoción de la Especialidad en Cuidados Intensivos de Obstetricia como los propone De León. ${ }^{17}$ Así como también apoyar a la Asociación de Medicina Critica en Obstetricia, mediante trabajos de investigación en esta área.

\section{AGRADECIMIENTOS}

Agradecemos a la dirección general del Hospital General de Matamoros, Tamaulipas y, en especial, a la Dra. Irlanda Peña, por sus finas atenciones y todas las facilidades prestadas para la realización del presente trabajo.

\section{BIBLIOGRAFÍA}

1. Wunsch H, Guerra C, Barnato AE, Angus DC, Li G, LindeZwirble WT. Three-year outcomes for medicare beneficiaries who survive intensive care. JAMA. 2010;303:849-856.

2. Derom R, Patel NB, Thiery M. Implications of increasing rates of cesarean section. Prog Obstet Gynecol. 1987;6:175-194.

3. Hall MH. Maternal mortality higher after cesarean section. BMJ. 1994;308:654-655.

4. Sachs BP, Yeh J, Acker D, et al. Cesarean section-related maternal mortality in Massachusetts, 1954-1985. Obstet Gynecol. 1988;71:385-388.

5. Campos-Castillo C, Hurtado-López M, Pulido-Reynosa A, Guerrero-Hernández A, Briones-Garduño JC. "Capítulo 1: Muerte materna en México". En: Briones-Garduño JC, Díaz de León Ponce M. Mortalidad materna. México, Editorial Alfil; 2013.

6. Fajardo-Dolci G, Meljem-Moctezuma J, Vicente-González E, Venegas-Páez FV, Villalba-Espinoza I, Pérez-Cardoso AL, et al. Análisis de las muertes maternas en México ocurridas durante 2009. Rev Med Inst Mex Seguro Soc. 2013;51(5):486-495.

7. Loverro G, Pansini V, Greco P, Vimercati A, Parisi AM, Selvaggi $L$. Indications and outcome for intensive care unit admission during puerperium. Arch Gynecol Obstet. 2001;265:195-198.

8. Lapinsky SE, Kruczynski K, Seaward GR, Farine D, Grossman RF. Critical care management of the obstetrics patient. Can J Anaesth. 1997;44:325-329.

9. Tang LC, Kwock AC, Wong AY, Lee YY, Sun KO, So AP. Critical care in obstetrical patients: an eight-year review. Chin Med $J$ (Engl). 1997;110:936-941.

10. Balderas-Peña LM, Canales-Muñoz JL, Angulo-Vázquez J, Anaya-Prado R, González Ojeda A. Síndrome HELLP ¿Evidencias de una posible respuesta inflamatoria sistémica en la Pre eclampsia? Ginecol Obstet Mex. 2002;70:328-337.

11. Tebeu PM, Halle-Ekane G, Da Itambi M, Enow Mbu R, Mawamba $\mathrm{Y}$, Fomulu JN. Maternal mortality in Cameroon: a university teaching hospital report. Pan Afr Med J. 2015;21:16.

12. Kazi S. Emergency peripartum hysterectomy: A great obstetric challenge. Pak J Med Sci. 2018;34(6):1567-1570.

13. Briones GJ, Díaz de León PM, Meneses CJ. Estrategias para reducir la mortalidad materna hospitalaria en el Estado de México. Rev Asoc Mex Med Crit Ter Int. 2009;11:16-24.

14. Guerrero-Hernández A, Briones-Vega CG. de León Ponce MD, Briones-Garduño JC. Cuidados intensivos en ginecología y obstetricia en el Hospital General de México. Rev Asoc Mex Med Crít Ter Int. 2011;25(4):211-217.

15. Gayet C, Gutiérrez JP. Calendario de inicio sexual en México. Comparación entre encuestas nacionales y tendencias en el tiempo. Salud Pública Mex. 2014;56(6):638-649.

16. UNICEF. La estrategia nacional para disminuir la mortalidad materna es un avance significativo para garantizar una maternidad segura. México, UNICEF; 2009.

17. Díaz de León-Ponce MA, Briones-Garduño JC. Medicina crítica en obstetricia (una verdad no reconocida). Rev Asoc Mex Med Crit y Ter Int. 2012;26(1):6-10.

Correspondencia:

Jesús Adrián Maldonado Mancillas

Hospital General de Matamoros Tamaulipas.

Universidad Autónoma de Tamaulipas,

Facultad de Medicina Matamoros.

Carretera Sendero Nacional $\mathrm{Km} 3$,

Matamoros, Tamaulipas, México.

Fax: (868) 8204-4000

E-mail: amaldona@uat.edu.mx 